\title{
Title: More than just oil droplets in water: surface tension and viscosity of protein condensates quantified by micropipette aspiration
}

\begin{abstract}
Authors: Huan Wang ${ }^{1}$, Fleurie M. Kelley², Dragomir Milovanovic ${ }^{3}$, Benjamin S. Schuster ${ }^{2}$, Zheng Shi ${ }^{1 *}$

Departments of ${ }^{1}$ Chemistry and Chemical Biology, ${ }^{2}$ Chemical and Biochemical Engineering, Rutgers University, Piscataway, New Jersey 08854, United States. ${ }^{3}$ Laboratory of Molecular Neuroscience, German Center for Neurodegenerative Diseases (DZNE), Berlin 10117, Germany
\end{abstract}

*Correspondence should be addressed to: zheng.shi@rutgers.edu

\section{Abstract:}

The material properties of biomolecular condensates play pivotal roles in many biological and pathological processes. Despite the rapid increase in the number of biomolecules identified that undergo liquid-liquid phase separation (LLPS), quantitative studies of the resulting condensates have been severely lagging behind. Here, we develop a micropipette-based technique, which uniquely allows quantifications of both the surface tension and viscosity of biomolecular condensates, independent of labeling and surface wetting effects. We demonstrate the accuracy and versatility of this technique by measuring condensates of LAF-1 RGG domains and a polymer-based aqueous two-phase system (ATPS). We anticipate this technique will be widely applicable to biomolecular condensates and will resolve several limitations regarding current approaches.

\section{Main Text}

Biomolecular condensates that arise from LLPS have recently emerged as a central player in numerous cellular processes ${ }^{1,2}$. Surface tension and viscosity are two independent parameters that define the material properties of a liquid ${ }^{3,4}$. Gradual increases in the viscosities of biomolecular condensates are often linked to the formation of fibrils that underlie aging-associated diseases $^{5-10}$. Quantification of condensate rheology therefore holds promise for unravelling the mechanisms, as well as facilitating therapeutic advances in the treatment of these diseases ${ }^{11}$.

While changes in condensate viscosity often have pathological consequences, the surface tension of biomolecular condensates can play key physiological roles: differences in surface tension can lead to layered multi-phase condensates, such as the compartmentation in nucleoli ${ }^{12-}$ 14. During autophagy, surface tension determines whether p62 condensates will be sequestered in small droplets or digested as a whole ${ }^{15}$. Finally, the nucleation of microtubule branches relies on an instability of TPX2 condensates, driven solely by the condensates' surface tension ${ }^{16}$.

Several techniques have been developed to probe either the viscosity or the surface tension of biomolecular condensates ${ }^{12,17-21}$. The most widely used measure of viscosity relies on fluorescence recovery after photobleaching (FRAP), which is challenging to quantify in the scenario of 3-dimensional compartments such as biomolecular condensates ${ }^{4,22}$. Measurements of surface tension rely heavily on the fusion kinetics between two condenstates ${ }^{17}$. While significant improvements have been made ${ }^{23,24}$, the fusion assay is intrinsically limited because only a ratio of surface tension to viscosity can be estimated ${ }^{12}$. Therefore, a user-friendly technique that can directly measure both surface tension and viscosity of biomolecular condensates is still missing.

Micropipette aspiration (MPA) has been well-established to study the elastic properties of cells and liposomes ${ }^{25,26}$. However, it has been challenging to apply MPA to quantify liquids. For MPA 
to perform well, the viscosity of the liquid needs to be large for the flow process to be adequately captured. At the same time, the surface tension of the liquid needs to be small for the aspiration pressure to overcome the capillary effect (see Methods). Both requirements are in contradiction to the properties of common liquids, where low viscosity $\left(10^{-3} \sim 10^{-2} \mathrm{~Pa} \cdot \mathrm{s}\right)$ and high surface tension $(\sim 10 \mathrm{mN} / \mathrm{m})$ are often observed. However, currently available data suggest that biomolecular condensates exhibit high viscosity ( $10 \mathrm{~Pa} \cdot \mathrm{s})$ and low surface tension $\left(10^{-3} \sim 10^{-2} \mathrm{mN} / \mathrm{m}\right)$, making them uniquely poised for quantitative MPA studies (Figure 1).

Here, we demonstrate the application of MPA to quantify the viscosity and surface tension of liquid condensates. We calibrated our method using a PEG-dextran ATPS ${ }^{27}$. This allowed us to develop a linear model to extract the material properties of condensates from their responses to MPA. We applied this technique to quantify condensates formed by the RGG domain, a wellknown RNA binding region of the $P$ granule RNA helicase LAF- $1^{18,28}$. We further confirmed our viscosity and surface tension measurements by FRAP and fusion assays, respectively. Our results suggest that material properties of protein condensates are closer to ATPS than to oil droplets in water. MPA represents an active microrheology technique that can simultaneously quantify independent properties of biomolecular condensates, insensitive to common sources of artifacts such as labeling, photobleaching, and wetting effects of proteins.

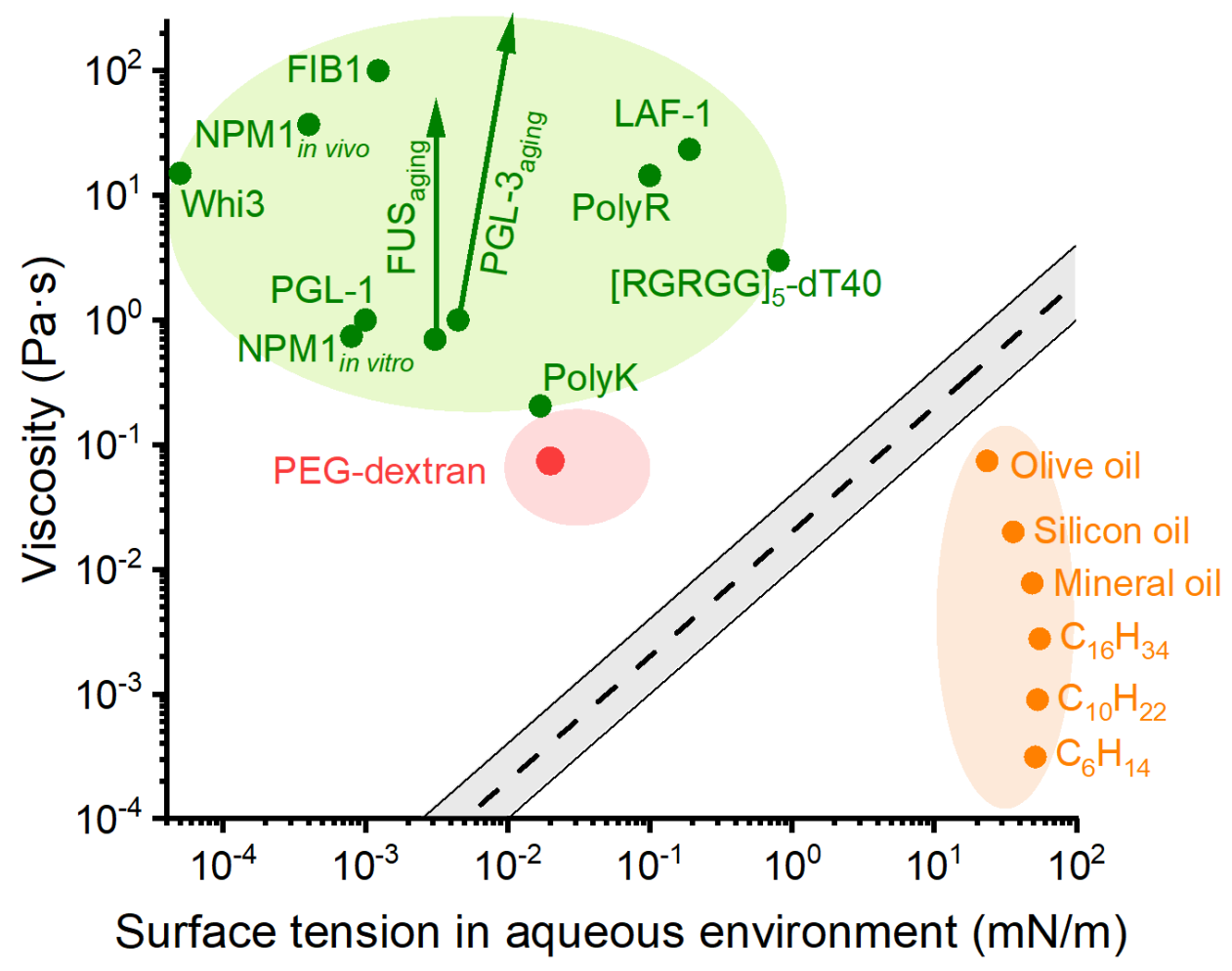

\section{Figure 1. Viscosity and surface tension of liquids.}

Viscosity and surface tension of biomolecular condensates in aqueous buffer (green, arrows represent changes of properties over time) and common 'oil droplets' in water (orange). The gray belt represents an estimated boundary above which MPA will be well-suited for viscosity and surface tension measurements (Methods). The red and pink regions represent values for dextranrich condensates measured in this study and estimated from literature ${ }^{29,30}$, respectively. See Table S1 for values and references used in this plot. 
To study the stress-strain relation of a liquid, a critical aspiration pressure $P_{\gamma}$, determined by the surface tension $(\gamma)$ of the condensate, needs to be reached. At aspiration pressures $\left(P_{\text {asp }}\right)$ greater than $P_{\gamma}$, the condensate will flow into the micropipette (Figure 2a, we define suction pressures as positive). For a Newtonian fluid, the pressure difference and the flow rate are linearly related via the condensate's viscosity $(\eta)$ :

$$
P_{\text {asp }}=M \cdot \eta \cdot V+P_{\gamma}
$$

Here, $V=d\left(L_{\mathrm{p}} / R_{\mathrm{p}}\right) / d t$ is the normalized flow rate. The critical pressure $P_{\gamma}=2 \gamma\left(H-1 / R_{c}\right)$. $L_{\mathrm{p}}, R_{\mathrm{p}}, R_{\mathrm{c}}, H$ describe the shape of the aspirated condensate and are readily available through microscopy (Figure 2a). However, the unitless factor $M$, which is a constant when $\left(R_{\mathrm{p}} / R_{\mathrm{c}}\right)^{3}<<1$, must be determined experimentally by aspirating liquids of known viscosities ${ }^{31}$. Then, by measuring $V$ under different $P_{\text {asp }}$, the viscosity and surface tension of an unknown liquid condensate can be quantified from the slope and intercept, respectively, of eq. 1.

To calibrate ' $M$ with liquids that are appropriate for MPA (Figure 1), we chose an ATPS composed of PEG (8000 Da) and dextran $(500,000 \mathrm{Da})^{15,29,32}$. Under a range of concentrations, mixtures of PEG and dextran will phase-separate into emulsions of micrometer-size droplets (Figure S1a). Rhodamine-B was included to identify the condensates microscopically. This mixture produced a labeled emulsion that is stable on the timescale of MPA experiments ( 10 min), but undergoes bulk phase separation after 1 2 hours (Figure 2b).

Stepwise aspiration pressures were applied to dextran-rich condensates, and the aspiration length was found to change linearly under each pressure step (Figure 2c, Figure S1b). The resulting relation between the aspiration pressure and the condensate flow rate (Figure 2d), agrees well with predictions of a Newtonian fluid. The slope $\mathrm{d} P_{\text {asp }} / \mathrm{d} V(37.0 \pm 0.7 \mathrm{~Pa} \cdot \mathrm{s} ; \mathrm{n}=6$; mean \pm SEM for all values reported herein), therefore, represents the viscosity of the dextran-rich phase multiplied by ' $M$ ' (eq.1). We then directly measured the viscosity by dragging an opticallytrapped particle within the dextran-rich phase (Figure 2e). The measured viscosity of the dextranrich phase (74 $\pm 4 \mathrm{mPa} \cdot \mathrm{s})$ agreed with bulk viscometer measurements (Methods), giving $M=500$ \pm 30 . Additionally, the intercept from the $P_{\text {asp }} V$ relation corresponds to a surface tension of 0.02 $\pm 0.01 \mathrm{mN} / \mathrm{m}$ (eq. 1), in agreement with the literature ${ }^{29}$. With careful control of water evaporation (Figure S2), next we applied MPA to protein samples of limited volumes $(20 \sim 30 \mu \mathrm{L})$. 
a

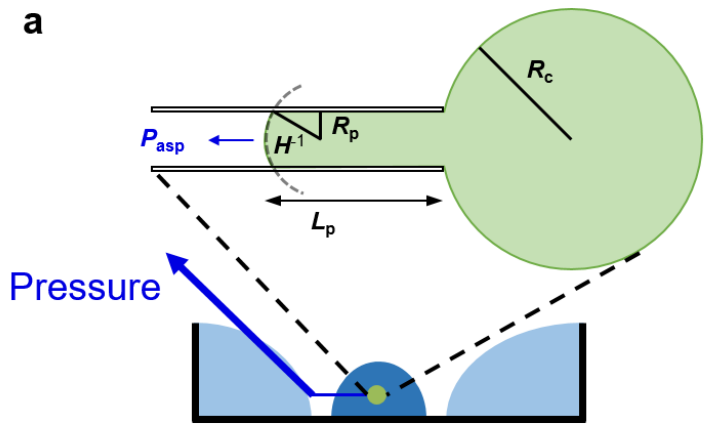

b Freshly mixed emulsion

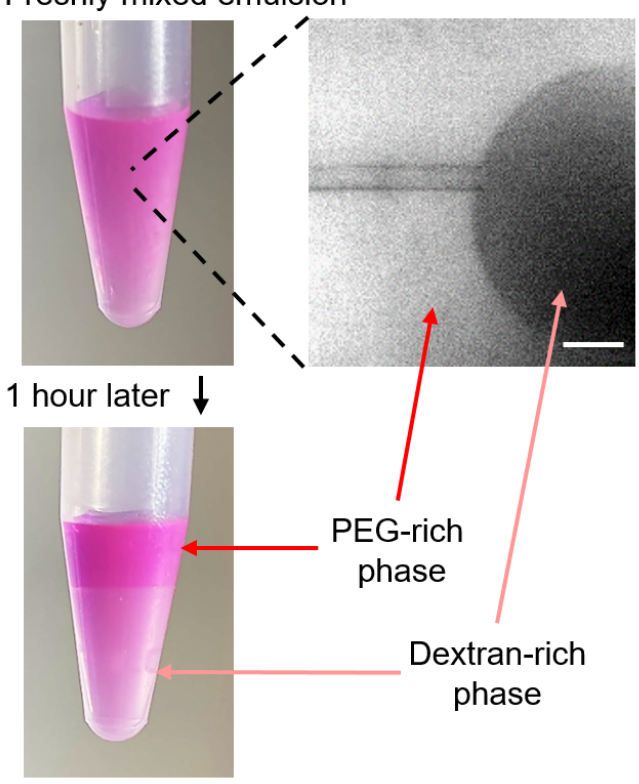

c
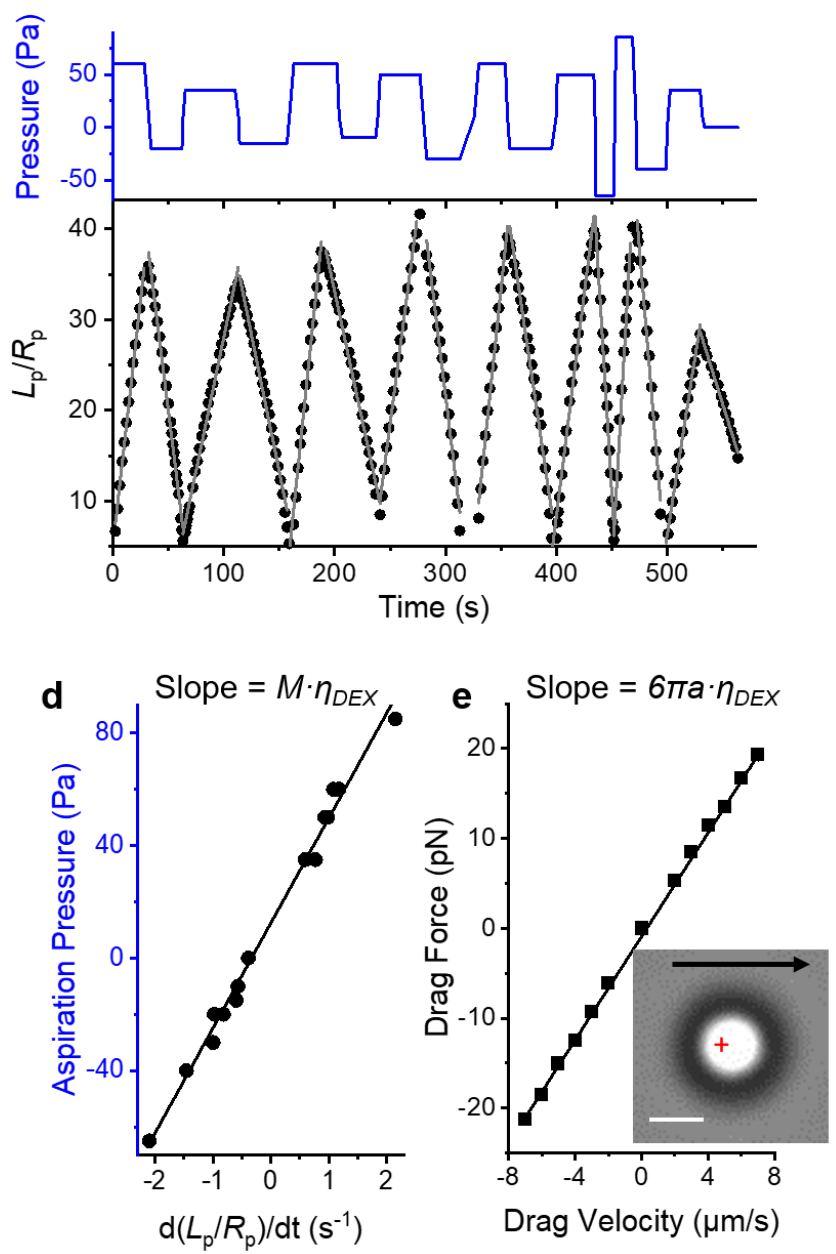

Figure 2. Calibration of MPA with PEG-dextran ATPS.

a, Illustration of the micropipette aspiration system, dark blue: sample, light blue: water, green: a pipette-aspirated condensate, $P_{\text {asp }}$ : aspiration pressure, $R_{\mathrm{p}}$ : pipette radius, $L_{\mathrm{p}}$ : aspiration length, $R_{\mathrm{c}}$ : radius of condensate outside the pipette. $H$ : mean curvature of the liquid interface in the micropipette (positive direction as illustrated). b. An emulsion of PEG-dextran (upper) undergoes bulk LLPS after creaming for 1 hour (lower). Scale bar, $20 \mu \mathrm{m}$. c, Aspiration pressure (upper) and normalized aspiration length (lower) during MPA. Gray lines: linear fits of the normalized aspiration length for each pressure step. d, $P_{\text {asp }}$ of each step plotted against $V$ (slopes of the gray lines in c). e, Viscosity determination by optical dragging. Inset image: a trapped polystyrene particle dragged at $5 \mu \mathrm{m} / \mathrm{s}$ (arrow) in the dextran-rich phase. Cross: trap-center. Scale bar, $2 \mu \mathrm{m}$. Linear fits: $R^{2}=0.990$ for $\mathbf{d}$ and 0.999 for $\mathbf{e}$.

LAF-1 is one of the first well-studied proteins that undergo LLPS, mainly due to its intrinsically disordered N-terminal RGG domain 4,18,19,22,28. We applied MPA to an engineered tandem RGG domain that robustly undergoes LLPS (Methods; hereafter named RGG condensates) ${ }^{28}$. Unlike dextran, RGG condensates fully wet the inner wall of the micropipette, requiring a negative $P_{\mathrm{y}}$ to balance the capillary effect (Figure $3 \mathrm{a}, H \approx-1 / R_{\mathrm{p}}$ ). Beyond $P_{\mathrm{V}}$, the aspiration length changed linearly under each pressure step (Figure 3b, Movie S1), indicating a lack of condensate elasticity at the timescale we were probing $(>1 \mathrm{~s})$. After initial-entry steps, $V$ increased linearly with $P_{\text {asp }}$ 
(Figure 3c, S3, S4). The slope corresponded to a viscosity of $1.62 \pm 0.18 \mathrm{~Pa} \cdot \mathrm{S}(\mathrm{n}=11)$, in agreement with previous estimates ${ }^{33}$. As expected from the wetting behavior, the intercept of $P_{\text {asp }}$ vs. $V$ was negative, and the corresponding surface tension was $0.159 \pm 0.010 \mathrm{mN} / \mathrm{m}(\mathrm{n}=11)$.

Many proteins tend to wet and adsorb onto solid surfaces ${ }^{4,16,23,24,34}$. While potentially mediating important biological processes ${ }^{15,16,34}$, this wetting effect can introduce significant artifacts in measurements that rely on the fusion kinetics or morphology of condensates ${ }^{12,23,24}$. In contrast, the contribution of $R_{\mathrm{c}}$ in eq. 1 is negligible when $R_{\mathrm{c}}{ }^{-1}<<|H|$, making MPA insensitive to the wetting of condensates (Figure 3c, Movie S2). Additionally, fluorescent labeling of the protein is not necessary for MPA as long as the condensate-buffer interfaces can be resolved (Figure S1c, S4, and Movie S3). For the same reasons, MPA measurements are insensitive to photobleaching and can be easily combined with fluorescence-based studies ${ }^{24,35}$, further expanding the applicability of this technique.

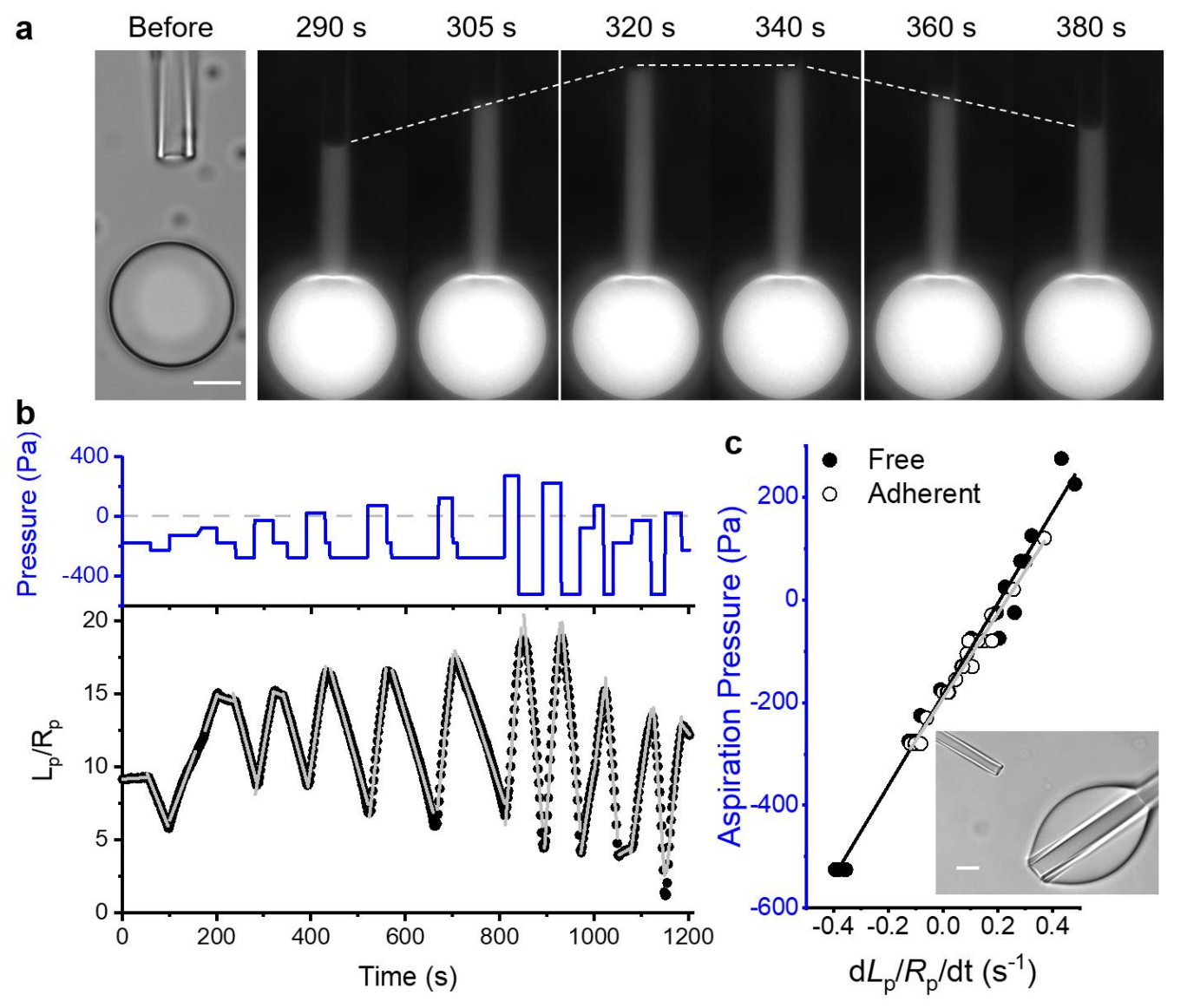

\section{Figure 3. MPA of RGG condensates.}

a, Left: transmitted light image of an RGG condensate with a nearby micropipette. Right: time lapse fluorescent images of the RGG condensate under three aspiration pressures: $-25 \mathrm{~Pa}$ (290 320 s), -175 Pa (320 340 s), and -275 Pa (340 380 s). Dashed lines trace the change of $L_{p}$. b. Aspiration pressure (upper) and normalized aspiration length (lower) during MPA. Dashed line: zero pressure. Gray lines: linear fits of the normalized aspiration length for each pressure step. c, $P_{\text {asp }}$ of each step plotted against $V$ for a free condensate (closed, see a) and a condensate strongly adhered to a glass pipette (open, inset image, see Methods). Linear fits to the data are 
shown as black (slope: $890 \pm 30 \mathrm{~Pa} \cdot \mathrm{s}$, intercept: $-184 \pm 7 \mathrm{~Pa}, \mathrm{R}^{2}=0.982$ ) and gray (slope: $830 \pm$ $30 \mathrm{~Pa} \cdot \mathrm{s}$, intercept: $-192 \pm 4 \mathrm{~Pa}, \mathrm{R}^{2}=0.976$ ) lines, respectively. All scale bars, $10 \mu \mathrm{m}$.

To confirm the surface tension and viscosity of RGG condensates measured by MPA, we first adopted an improved version of the condensate fusion assay ${ }^{23,24}$. Two optically-trapped RGG condensates were manipulated to encounter and the subsequent fusion process was recorded (Figure 4a, 4b). A linear relation was observed between the fusion time and the size of the condensates (Figure 4c). The slope, which scales with the inverse capillary velocity $\eta / \gamma$, was 0.016 $\pm 0.002 \mathrm{~s} / \mu \mathrm{m}$, in agreement with the MPA measurements $(\eta / \gamma=0.010 \pm 0.001 \mathrm{~s} / \mu \mathrm{m})$. We then used FRAP to estimate the viscosity of RGG condensates. A circular region within RGG condensates was photobleached, and diffusion coefficients were calculated based on the halfrecovery time (Figure $4 \mathrm{~d})^{22}$. Combined with an estimate of the protein hydrodynamic radius, we obtained a viscosity of $1.8 \mathrm{~Pa} \cdot \mathrm{s}$, comparable to the MPA result. We noticed that viscosity values between 0.8 and 3.6 Pa.s can be extracted from FRAP, depending on the extract model of choice (Methods, Figure 4e).

a

1)

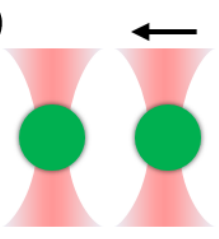

3)

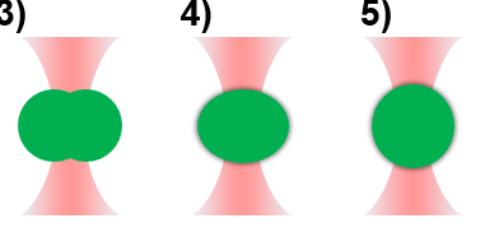

2)

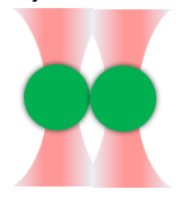

5) b

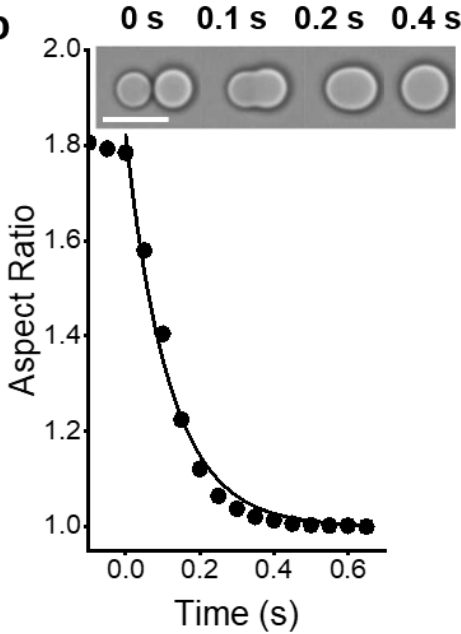

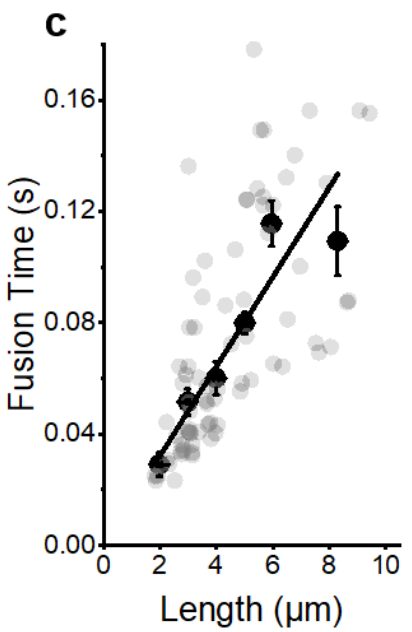
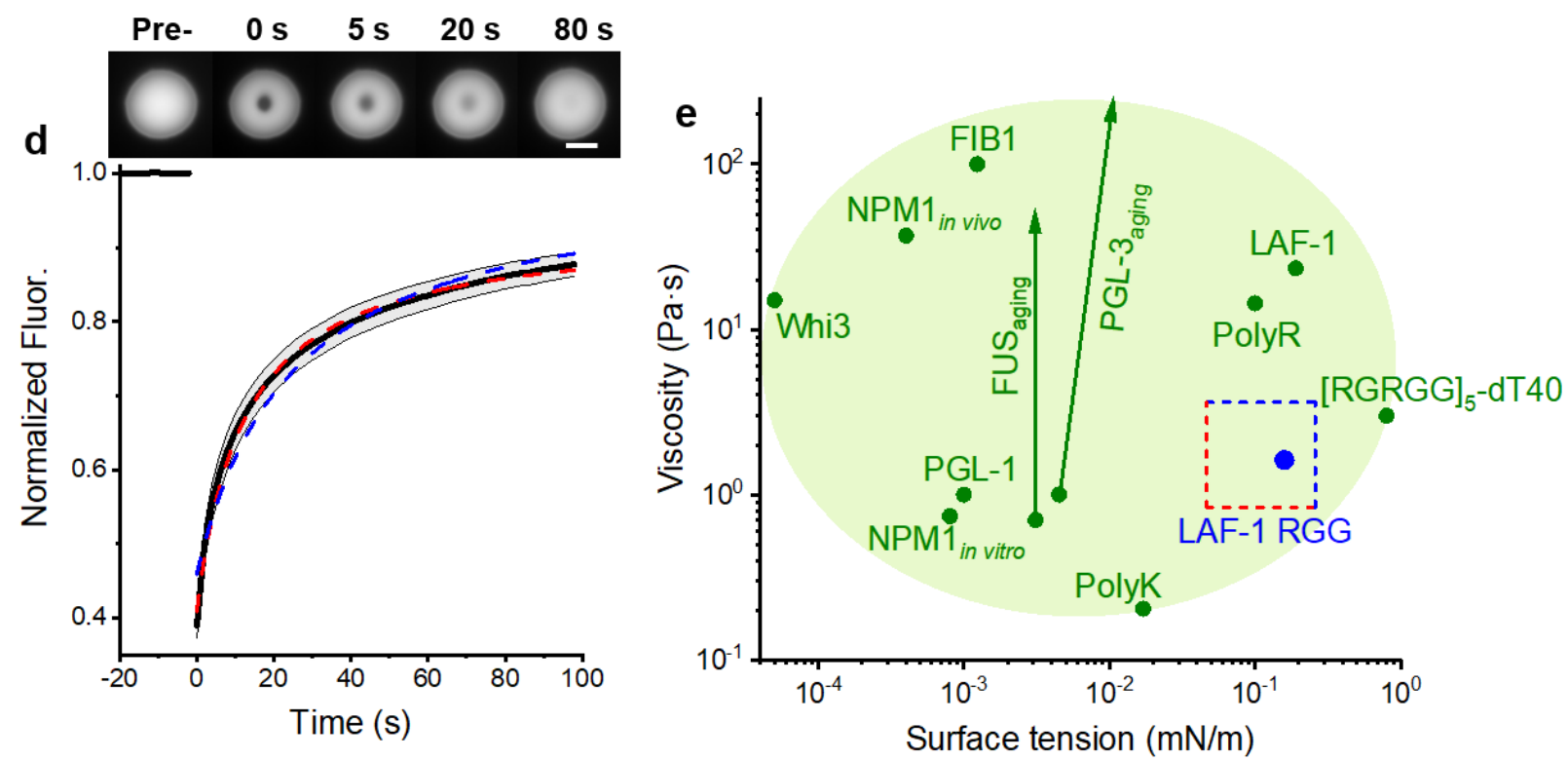
Figure 4. Surface tension and viscosity of RGG condensates estimated from condensate fusion and FRAP.

a, 1)-5): Illustration of the condensate fusion experiment using dual-optical traps. $\mathbf{b}$, Fusion of two RGG condensates (inset images) quantified as a decrease of the overall aspect ratio to 1 . The curve is an exponential fit. c, The fusion time of RGG condensates vs. the condensates' length. Gray and black circles are individual ( $n=76$ pairs) and binned fusion experiments, respectively. Line: weighted linear fit to the binned data (slope $=0.016 \pm 0.002 \mathrm{~s} / \mu \mathrm{m}, \mathrm{R}^{2}=0.902$ ). $d$, FRAP measurements within RGG condensates $(n=42)$. Inset images show a representative experiment. Red dash: fit to $I(t)=\frac{I_{0}+I_{\infty} \frac{t}{\tau_{1 / 2}}}{1+\frac{t}{\tau_{1 / 2}}}$, with $\tau_{1 / 2}=12.4 \pm 0.5 \mathrm{~s}$ and $I_{\infty}=0.928 \pm 0.003, \mathrm{R}^{2}=$ 0.995. Blue dash: fit to $I(t)=\frac{I_{0}+\frac{t}{\tau_{1 / 2}}}{1+\frac{t}{\tau_{1 / 2}}}$, with $\tau_{1 / 2}=24 \pm 1 \mathrm{~s}, \mathrm{R}^{2}=0.966$. e, Zoom-in of Figure 1, with viscosity and surface tension of RGG condensates measured by MPA in blue circle. Estimates from FRAP and fusion kinetics are represented by the dished box (Methods). All error bars are SEM, all scale bars are $10 \mu \mathrm{m}$.

RGG condensates fuse quickly $(\sim 0.1 \mathrm{~s})$ with $>90 \%$ of the constituting proteins moving freely, consistent with their liquid behavior during MPA. However, many biomolecular condensates can take more than $100 \mathrm{~s}$ to fuse ${ }^{15,36}$, while exhibiting small fractions $(<50 \%)$ of mobile proteins ${ }^{3,10,37,38}$. In the latter cases, MPA measurements will be essential to clarify confusion around the condensates' material properties ${ }^{10}$. The unambiguous quantification of RGG condensates through MPA further iterates the contrasting material properties of biomolecular condensates and oil droplets (Figure 1). For example, mineral oil has a surface tension 300-fold higher than that of RGG condensates, whereas its viscosity is more than 200 -fold lower.

Surface tension and viscosity are tunable through intermolecular interactions. Thus, a future direction will be to systematically dissect how protein sequence and biochemical environment affect the material properties of biomolecular condensates ${ }^{4}$. Importantly, by implementing a whole-cell patch-clamp configuration ${ }^{39}$, MPA can be applied to study biomolecular condensates in vivo. Finally, MPA setups are readily available in electrophysiology and biomechanics labs, making it easily adaptable for studying the material properties of biomolecular condensates in the broader biological and chemical communities.

Acknowledgements: We want to thank Ehsan Atefi, Ningwei Li, Frank Jülicher, and Rick Remsing for helpful discussions. We thank Gayatri Ganeshan, Steven Arnold, and Roberto Sul for critical comments on the manuscript. We also thank Andy Nieuwkoop and his lab for helping with materials and storage space. Z.S. and B.S.S are supported by Rutgers University startup funds. F.M.K is supported by NIH Award T32 GM135141. D.M. is supported by the German Research Foundation (DFG MI 2104 and SFB 1286) and the German Academic Exchange Service (DAAD PPE 2021). 


\section{References}

1. Shin, Y.; Brangwynne, C. P. Liquid phase condensation in cell physiology and disease. Science 2017, 357.

2. Banani, S. F.; Lee, H. O.; Hyman, A. A.; Rosen, M. K. Biomolecular condensates: organizers of cellular biochemistry. Nature reviews Molecular cell biology 2017, 18, 285-298.

3. Bracha, D.; Walls, M. T.; Brangwynne, C. P. Probing and engineering liquid-phase organelles. Nat. Biotechnol. 2019, 1-11.

4. Schuster, B. S.; Regy, R. M.; Dolan, E. M.; Kanchi Ranganath, A.; Jovic, N.; Khare, S. D.; Shi, Z.; Mittal, J. Biomolecular Condensates: Sequence Determinants of Phase Separation, Microstructural Organization, Enzymatic Activity, and Material Properties. The Journal of Physical Chemistry B 2021.

5. Mathieu, C.; Pappu, R. V.; Taylor, J. P. Beyond aggregation: Pathological phase transitions in neurodegenerative disease. Science 2020, 370, 56-60.

6. Nedelsky, N. B.; Taylor, J. P. Bridging biophysics and neurology: aberrant phase transitions in neurodegenerative disease. Nature Reviews Neurology 2019, 15, 272-286.

7. Alberti, S.; Dormann, D. Liquid-liquid phase separation in disease. Annu. Rev. Genet. 2019, 53, 171-194.

8. Jawerth, L.; Fischer-Friedrich, E.; Saha, S.; Wang, J.; Franzmann, T.; Zhang, X.; Sachweh, J.; Ruer, M.; ljavi, M.; Saha, S.; Mahamid, J.; Hyman, A. A.; Jülicher, F. Protein condensates as aging Maxwell fluids. Science 2020, 370, 1317-1323.

9. Guo, L.; Kim, H. J.; Wang, H.; Monaghan, J.; Freyermuth, F.; Sung, J. C.; O’Donovan, K.; Fare, C. M.; Taylor, J. P.; Shorter, J. Nuclear-import receptors reverse aberrant phase transitions of RNA-binding proteins with prion-like domains. Cell 2018, 173, 677-692. e20.

10. Putnam, A.; Cassani, M.; Smith, J.; Seydoux, G. A gel phase promotes condensation of liquid $\mathrm{P}$ granules in Caenorhabditis elegans embryos. Nature structural \& molecular biology 2019, 26, 220-226.

11. Guo, L.; Fare, C. M.; Shorter, J. Therapeutic dissolution of aberrant phases by nuclearimport receptors. Trends Cell Biol. 2019, 29, 308-322.

12. Feric, M.; Vaidya, N.; Harmon, T. S.; Mitrea, D. M.; Zhu, L.; Richardson, T. M.; Kriwacki, R. W.; Pappu, R. V.; Brangwynne, C. P. Coexisting liquid phases underlie nucleolar subcompartments. Cell 2016, 165, 1686-1697.

13. Fisher, R. S.; Elbaum-Garfinkle, S. Tunable multiphase dynamics of arginine and lysine liquid condensates. Nature Communications 2020, 11, 1-10. 
14. Yu, H.; Lu, S.; Gasior, K.; Singh, D.; Vazquez-Sanchez, S.; Tapia, O.; Toprani, D.; Beccari, M. S.; Yates, J. R.; Da Cruz, S.; Cleveland, D. W. HSP70 chaperones RNA-free TDP-43 into anisotropic intranuclear liquid spherical shells. Science 2021, 371.

15. Agudo-Canalejo, J.; Schultz, S. W.; Chino, H.; Migliano, S. M.; Saito, C.; Koyama-Honda, I.; Stenmark, H.; Brech, A.; May, A. I.; Mizushima, N.; Knorr, R. L. Wetting regulates autophagy of phase-separated compartments and the cytosol. Nature 2021, 591, 142-146.

16. Setru, S. U.; Gouveia, B.; Alfaro-Aco, R.; Shaevitz, J. W.; Stone, H. A.; Petry, S. A hydrodynamic instability drives protein droplet formation on microtubules to nucleate branches. Nature Physics 2021, 1-6.

17. Brangwynne, C. P.; Eckmann, C. R.; Courson, D. S.; Rybarska, A.; Hoege, C.; Gharakhani, J.; Jülicher, F.; Hyman, A. A. Germline P granules are liquid droplets that localize by controlled dissolution/condensation. Science 2009, 324, 1729-1732.

18. Elbaum-Garfinkle, S.; Kim, Y.; Szczepaniak, K.; Chen, C. C.; Eckmann, C. R.; Myong, S.; Brangwynne, C. P. The disordered $\mathrm{P}$ granule protein LAF-1 drives phase separation into droplets with tunable viscosity and dynamics. Proceedings of the National Academy of Sciences 2015, 112, 7189-7194.

19. Taylor, N.; Elbaum-Garfinkle, S.; Vaidya, N.; Zhang, H.; Stone, H. A.; Brangwynne, C. P. Biophysical characterization of organelle-based RNA/protein liquid phases using microfluidics. Soft matter 2016, 12, 9142-9150.

20. Jawerth, L. M.; ljavi, M.; Ruer, M.; Saha, S.; Jahnel, M.; Hyman, A. A.; Jülicher, F.; FischerFriedrich, E. Salt-dependent rheology and surface tension of protein condensates using optical traps. Phys. Rev. Lett. 2018, 121, 258101.

21. Caragine, C. M.; Haley, S. C.; Zidovska, A. Surface fluctuations and coalescence of nucleolar droplets in the human cell nucleus. Phys. Rev. Lett. 2018, 121, 148101.

22. Taylor, N. O.; Wei, M.; Stone, H. A.; Brangwynne, C. P. Quantifying dynamics in phaseseparated condensates using fluorescence recovery after photobleaching. Biophys. J. 2019, 117, 1285-1300.

23. Alshareedah, I.; Thurston, G. M.; Banerjee, P. R. Quantifying viscosity and surface tension of multicomponent protein-nucleic acid condensates. Biophys. J. 2021.

24. Ghosh, A.; Zhou, H. Determinants for Fusion Speed of Biomolecular Droplets. Angewandte Chemie International Edition 2020, 59, 20837-20840.

25. Shi, Z.; Baumgart, T. Membrane tension and peripheral protein density mediate membrane shape transitions. Nature communications 2015, 6, 1-8.

26. Hochmuth, R. M. Micropipette aspiration of living cells. J. Biomech. 2000, 33, 15-22.

27. Crowe, C. D.; Keating, C. D. Liquid-liquid phase separation in artificial cells. Interface Focus 2018, 8, 20180032. 
28. Schuster, B. S.; Reed, E. H.; Parthasarathy, R.; Jahnke, C. N.; Caldwell, R. M.; Bermudez, J. G.; Ramage, H.; Good, M. C.; Hammer, D. A. Controllable protein phase separation and modular recruitment to form responsive membraneless organelles. Nature communications 2018, 9, 1-12.

29. Atefi, E.; Mann Jr, J. A.; Tavana, H. Ultralow interfacial tensions of aqueous two-phase systems measured using drop shape. Langmuir 2014, 30, 9691-9699.

30. Carrasco, F.; Chornet, E.; Overend, R. P.; Costa, J. A generalized correlation for the viscosity of dextrans in aqueous solutions as a function of temperature, concentration, and molecular weight at low shear rates. J Appl Polym Sci 1989, 37, 2087-2098.

31. Drury, J. L.; Dembo, M. Hydrodynamics of micropipette aspiration. Biophys. J. 1999, 76, 110-128.

32. Helfrich, M. R.; Mangeney-Slavin, L. K.; Long, M. S.; Djoko, K. Y.; Keating, C. D. Aqueous phase separation in giant vesicles. J. Am. Chem. Soc. 2002, 124, 13374-13375.

33. Schuster, B. S.; Dignon, G. L.; Tang, W. S.; Kelley, F. M.; Ranganath, A. K.; Jahnke, C. N.; Simpkins, A. G.; Regy, R. M.; Hammer, D. A.; Good, M. C. Identifying sequence perturbations to an intrinsically disordered protein that determine its phase-separation behavior. Proceedings of the National Academy of Sciences 2020, 117, 11421-11431.

34. Su, X.; Ditlev, J. A.; Hui, E.; Xing, W.; Banjade, S.; Okrut, J.; King, D. S.; Taunton, J.; Rosen, M. K.; Vale, R. D. Phase separation of signaling molecules promotes T cell receptor signal transduction. Science 2016, 352, 595-599.

35. Alberti, S.; Gladfelter, A.; Mittag, T. Considerations and challenges in studying liquid-liquid phase separation and biomolecular condensates. Cell 2019, 176, 419-434.

36. Zhang, H.; Elbaum-Garfinkle, S.; Langdon, E. M.; Taylor, N.; Occhipinti, P.; Bridges, A. A.; Brangwynne, C. P.; Gladfelter, A. S. RNA controls PolyQ protein phase transitions. Mol. Cell 2015, 60, 220-230.

37. Hoffmann, C.; Sansevrino, R.; Morabito, G.; Logan, C.; Vabulas, R. M.; Ulusoy, A.; Ganzella, M.; Milovanovic, D. Synapsin Condensates Recruit alpha-Synuclein. J. Mol. Biol. 2021, 166961.

38. Ghosh, A.; Zhang, X.; Zhou, H. Tug of war between condensate phases in a minimal macromolecular system. J. Am. Chem. Soc. 2020, 142, 8848-8861.

39. Zhang, H.; Reichert, E.; Cohen, A. E. Optical electrophysiology for probing function and pharmacology of voltage-gated ion channels. Elife 2016, 5, e15202. 


\title{
Title: More than just oil droplets in water: surface tension and viscosity of protein condensates quantified by micropipette aspiration
}

\author{
Authors: Huan Wang ${ }^{1}$, Fleurie M. Kelley², Dragomir Milovanovic ${ }^{3}$, Benjamin S. Schuster², \\ Zheng Shi ${ }^{1 *}$ \\ Departments of ${ }^{1}$ Chemistry and Chemical Biology, ${ }^{2}$ Chemical and Biochemical Engineering, Rutgers \\ University, Piscataway, New Jersey 08854, United States. ${ }^{3}$ Laboratory of Molecular Neuroscience, \\ German Center for Neurodegenerative Diseases (DZNE), Berlin 10117, Germany \\ *Correspondence should be addressed to: zheng.shi@rutgers.edu
}

\section{Supplementary information includes:}

\author{
Methods \\ Supplementary Figures S1-S4 \\ Supplementary Movies S1-S3 \\ Supplementary Table S1
}




\section{Methods}

Estimate of the working range of micropipette aspiration (MPA) through dimensional analysis

During micropipette aspiration of a liquid (Figure 2a), the viscosity needs to be large in order for the camera to capture the flow process. At the same time, the surface tension needs to be small in order for the flow to start.

Assume the maximal imaging frequency is $100 \mathrm{~Hz}\left(\Delta t_{\min }=0.01 \mathrm{~s}\right)$, the radius of the pipette: $R_{p}=$ $1 \mu \mathrm{m}$, and $M=500$ (see eq. 1 in the main text). In order to capture liquid deformations that are on the order of pipette diameter $\left(\Delta L_{p}=2 \mu \mathrm{m}\right)$, the viscosity $\eta$ (in Pa.s) needs to satisfy:

$$
\eta>\left|P_{\mathrm{asp}}\right| \cdot \frac{R_{\mathrm{p}} \Delta t_{\mathrm{min}}}{M \Delta L_{\mathrm{p}}}=10^{-5}\left|P_{\mathrm{asp}}\right|
$$

The aspiration pressure needs to overcome the capillary effect caused by the surface tension $Y$ (in $\mathrm{mN} / \mathrm{m})$. For a non-wetting $\left(H^{-1}=R_{\mathrm{p}}\right)$ or a perfectly wetting $\left(H^{-1}=-R_{\mathrm{p}}\right)$ liquid with $R_{\mathrm{c}}>>R_{\mathrm{p}}$ :

$$
\left|P_{\text {asp }}\right|>\frac{2 \gamma}{R_{\mathrm{p}}}=2 \times 10^{3} \gamma
$$

Combine relations $\mathrm{S} 1$ and $\mathrm{S} 2$ :

$$
\eta(\text { in } \mathrm{Pa} \cdot \mathrm{s})>2 \times 10^{-2} \gamma(\text { in } \mathrm{mN} / \mathrm{m})
$$

Therefore, relation S3 defines the regime of viscosity and surface tension where MPA is expected to perform well. In Figure 1, $\eta=0.02 \gamma$ is plotted as the black dashed line, gray regions represent $\eta=0.01 \gamma \sim 0.04 \gamma$.

\section{Protein purification and sample preparation}

RGG-based proteins were expressed recombinantly in $E$. coli and purified by affinity chromatography, as previously described". The working protein sample contains $1 \mu \mathrm{M}$ RGGEGFP-RGG (molecular mass $62.1 \mathrm{kDa}$ ) and $6 \mu$ M RGG-RGG (molecular mass $35.7 \mathrm{kDa}$ ) in a pH 7.5 buffer containing $20 \mathrm{mM}$ Tris and $150 \mathrm{mM} \mathrm{NaCl}$.

Phase separated dextran and PEG aqueous two-phase systems were prepared by mixing different concentrations of PEG-8000 (43443-22, Alfa Aesar, US) and dextran-500k (DE132100GM, Spectrum Chemical, US) stock solutions. The stock solutions were prepared by dissolving each polymer in Milli-Q water. Emulsions of different PEG to dextran ratios showed different distributions of droplet size (Figure S1a). The 5\% PEG and 6.4\% dextran (both by mass) mixture was chosen for micropipette aspiration, because the resulting emulsion contained droplets with comparable sizes to those of the protein condensates.

Rhodamine-B (83689-1G, Sigma, USA) was added (at a final concentration of $1 \mu \mathrm{M}$ ) to the PEGdextran mixture to distinguish the dextran phase from the PEG phase (Figure $2 b$ ). Rhodamine-B preferentially enters the PEG-rich phase ${ }^{2}$, therefore dextran-rich condensates showed as dark droplets in a bright background in fluorescent microscopy images (Figure 2b, upper right image). The fluorescent labeling was confirmed by the observation that after bulk LLPS, the heavier dextran-rich layer (Figure $2 \mathrm{~b}$, lower layer of the lower left image) contained less Rhodamine-B compared to the lighter PEG-rich layer. The concentration of dextran in the dextran-rich layer was estimated to be $\sim 14 \%$ by mass. 
Micropipette fabrication, aspiration, and imaging

Micropipettes were pulled from glass capillaries using a pipette puller (PUL-1000, World Precision Instruments (WPI), US). The tip of the pipette was cut to an opening diameter between $1 \sim 5 \mu \mathrm{m}$ and bent to $\sim 40^{\circ}$ using a microforge (DMF1000, WPI).

Micropipette aspiration and imaging were carried out on a Ti2-A inverted fluorescent microscope (Nikon, Japan) equipped with a motorized stage and two motorized 4-axes micromanipulators (PatchPro-5000, Scientifica, UK). A micropipette was filled with the same buffer as the protein (20 $\mathrm{mM}$ Tris and $150 \mathrm{mM} \mathrm{NaCl}, \mathrm{pH}$ 7.5) using a MICROFIL needle (WPI) and subsequently mounted onto a micromanipulator. The rear end of the pipette was connected to an adjustable water reservoir. The pipette holder was then rotated so that the bent tip of the micropipette was parallel to the imaging plane. The aspiration pressure within the micropipette was controlled and recorded by adjusting the water level in the reservoir using a set of $5 \mathrm{ml}, 20 \mathrm{ml}, 50 \mathrm{ml}$, and $150 \mathrm{ml}$ syringes connected to the reservoir.

The zero pressure of the system was calibrated before each MPA experiment, using a dilute solution of fluorescent nanoparticles. The zero pressure $\left(P_{0}\right)$ was set according to the point where fluorescent nanoparticles underwent Brownian motion inside the micropipette. The error in aspiration pressure $(<2 \mathrm{~Pa})$ was defined as the minimal pressure change near $P_{0}$ that resulted in an observable directed flow of fluorescent particles in the micropipette.

MPA experiments were carried out in glass-bottom dishes (ES56291, Azer Scientific, US) that were pre-treated with 5\% Pluronic F127 (P2443-250G, Sigma) for > 1 hour to prevent adhesion of $R G G$ condensates to the glass ${ }^{1}$. Milli-Q water was added to the edge of the dish to minimize evaporation from the sample (Figure 2a). We further quantified water evaporation rates under our experimental conditions using a $20 \mu \mathrm{L}$ sample of Rhodamine-B solution (Figure S2). Volume of the sample was assumed to be inversely proportional to its mean fluorescence intensity. No measurable volume change was observed when the dish-cap was on, providing a stable environment for necessary incubation periods for the sample. When the dish-cap was removed for micropipette aspiration, evaporation led to a slow constant decrease in the sample volume $(\sim 0.04 \mu \mathrm{L} / \mathrm{min})$. We found that the evaporation can be compensated to be less than $5 \%$ in our MPA experiments (Figure S2).

After calibration of the aspiration pressure, a 20 30 $\mu \mathrm{L}$ sample of phase separated protein solution was added to the center of the dish (Figure 2a). Once micrometer-size protein condensates were observed at the bottom of the dish, a calibrated micropipette was moved to a condensate of interest to start the aspiration measurements. First, a positive (suction) pressure was applied to initiate the flow of the condensate into the micropipette. The condensate was typically allowed to flow into the micropipette until the aspiration length reached $\sim 40 \mu \mathrm{m}$ (the maximal aspiration length was limited by the field of view of the camera, initial condensate size, and the exact angle of the micropipette tip). Then, sequential stepwise ejection and suction pressures were applied to deform the condensate at different shear stresses while maintaining the aspiration length to be between 5 to $40 \mu \mathrm{m}$ (Figure $3 \mathrm{~b}$ and S3a-b). The deformation of the condensate was recorded using a 60X objective, at $1 \mathrm{~Hz}$ (ORCA-Flash 4.0, Hamamatsu, Japan), either through transmitted light imaging (Figure S1, S4, Movie S3) or through imaging the fluorescence of the EGFP tag (Figure 3, S3, Movies S1, S2). Of note, larger condensates typically result in more accurate MPA measurements, mainly due to the smaller perturbation of the changing aspiration length to $R_{\mathrm{c}}$. For a $>10 \mu \mathrm{m}$ condensate, typical changes in aspiration length 
correspond to a $<3 \%$ change in $R_{\mathrm{c}}$. Therefore, in our experiments, small condensates were first manipulated into a large condensate through either a micropipette or an optical trap (see "Optical trap mediated condensate fusion" section) before MPA measurements.

When the RGG condensate first entered the micropipette, wetting between protein and glass led to dramatic changes in the interfacial curvature between the condensate and buffer inside the micropipette. The interfacial curvature stabilized in later steps (Figure S3a, Movie S1). As a result, the $P_{\text {asp }}$ vs. $V$ relation during the initial-entry largely deviated from that of the remaining steps (Figure S3b, S3c). We corrected for the change in $\mathrm{H}$ by subtracting a time-dependent $P_{\mathrm{y}}$ from the aspiration pressure (Figure S3d). However, the irreversible binding of a trace amount of protein to the inner wall of the aspiration pipette significantly accelerated the deformation of condensates during the initial-entry steps (Figure S3e, S3f). To account for the lack of information about the kinetics of protein-glass binding, we disregarded the measurements from the initial-entry steps.

After the initial steps, the interfacial curvature between RGG condensates and buffer in the micropipette was set by the wetting of the protein to the inner pipette wall (Movie S1). Due to this wetting effect, RGG condensates flowed into the micropipette under both positive (suction) and small negative (ejection) pressures, whereas decreases in aspiration length only happened under large negative (ejection) pressures (Figure 3, Movie S1, Figure S4).

Adhesion between RGG condensates and the glass bottom dish can be prevented by coating the glass with $5 \%$ Pluronic F1271. However, we noticed that the same coating procedure does not necessarily work for other protein condensates or glass surfaces. To expand the applicability of MPA, we compared the measurements on near-free condensates that sedimented to the bottom of coated dishes (Figure 3a) with condensates that are purposefully adhered to a bare glass pipette (Figure 3c). MPA gave near identical $P_{\text {asp }}$ vs. $V$ relations for the two types of RGG condensates (Figure $3 \mathrm{c}$ ), even though $R_{\mathrm{c}}$ was not accurately defined in the strongly-adhered condensates. This agrees with expectations from eq. 1 , where the contribution of $R_{\mathrm{c}}$ can be neglected when $\left|R_{\mathrm{c}}{ }^{-1}\right|<<H$, as is in our experiments.

\section{Viscosity of dextran-rich condensates}

To calibrate the viscosity measurements, MPA should be applied to condensates with viscosity values that can be easily determined through other means. Dextran-rich condensates in a PEGdextran aqueous two-phase system were chosen for this purpose (Figure 2). After MPA, two independent methods were used to measure the viscosity of the dextran-rich phase.

1. Optical dragging

An optical trap (Tweez305, Aresis, Slovenia) was applied to drag an $r=1.60 \mu \mathrm{m}$ radius polystyrene bead (HUP-30-5, Spherotech, US) in a large dextran-rich condensate at 13 different velocities (Figure 2e). The slope of the dragging force $(f)$ vs. dragging speed $(v)$ was used to calculate the viscosity $(\eta)$ based on the Stokes equation (eq. S4):

$$
\frac{d f}{d v}=\eta \cdot 6 \pi r
$$

The measured viscosity was $74 \pm 4 \mathrm{mPa} \cdot \mathrm{s}$. The stiffness of the optical trap $(\sim 0.02 \mathrm{pN} / \mathrm{nm})$ was calibrated before each experiment by applying equipartition theorem to the thermal fluctuation of a trapped bead in the dextran-rich phase ${ }^{3}$.

2. Ubbelohde viscometer 
After the bulk-phase separation of $40 \mathrm{ml}$ PEG-dextran mixture, the bottom layer, corresponding to the dextran-rich phase, was applied through an Ubbelohde viscometer (13614C, Cannon Instrument, US). The viscosity was measured to be $80 \mathrm{mPa} \cdot \mathrm{s}$.

\section{Optical trap mediated condensate fusion}

Two RGG condensates were individually controlled by two independent optical traps (Tweez305, Aresis, Slovenia) equipped on the Ti2-A inverted microscope (Nikon, Japan). As illustrated in Figure $4 \mathrm{a}$, the right condensate was moved towards the left one until they touched. Then, the right optical trap was turned off, and the condensates were allowed to fuse under the combined influence of their viscosity and surface tension. The fusion processes were acquired at a frame rate of $20 \mathrm{~Hz}$ using a 60x water objective. The acquired images were analyzed in MATLAB (R2019a). The images were fitted into a Gaussian ellipse and the ratio of the major to minor axes of the ellipse (aspect ratio) was plotted as a function of time. The fusion time $(\tau)$ was extracted by fitting the change in the aspect ratio $(A R)$ of fusing condensates to a single exponential decay (Figure 4b, eq. S5).

$$
A R=1+\left(A R_{0}-1\right) \mathrm{e}^{-t / \tau}
$$

The length of condensates was defined as the geometric mean of the condensate diameters before fusion ${ }^{4}$. The ratio of viscosity to surface tension (inverse capillary velocity) was estimated from the slope of the fusion time vs. length relation (Figure 4c).

\section{FRAP measurement of the condensate viscosity}

FRAP experiments were performed on a total internal reflection fluorescence microscope (DMi8 TIRF, Leica, Germany) equipped with an Infinity Scanner system (Leica, Germany). All images were acquired using a $100 \mathrm{X}$ oil objective at $1 \mathrm{~Hz}$. A $1.5 \mu \mathrm{m}$ radius circular region was photobleached at the center of large RGG condensates (radius $9 \pm 2 \mu \mathrm{m})$ using a short pulse $(\sim 1$ s) of focused $488 \mathrm{~nm}$ laser, and the fluorescence recovery was analyzed using ImageJ. After background subtraction, fluorescence of the bleached region (/RoI) was divided by the fluorescence of the entire condensate ( $\left.I_{\text {cond }}\right)$ according to eq. S6, to minimize photobleaching and boundary effects ${ }^{5,6}$.

$$
I(t)=\frac{I_{R O I}(t)-I_{\text {background }}(t)}{I_{\text {cond }}(t)-I_{\text {background }}(t)}
$$

The time point right after the bleaching step was defined as time zero. $I(t)$ was normalized so that the average of $I(t<0)$ equals to 1 .

To extract the half-recovery time, $I(t)$ was fitted to eq. $S 7$ a or eq. $S 7 \mathrm{~b}$, depending on whether an immobile fraction was included in the model.

$$
\begin{aligned}
& I(t)=\frac{I_{0}+I_{\infty} \frac{t}{\tau_{1 / 2}}}{1+\frac{t}{\tau_{1 / 2}}} \\
& I(t)=\frac{I_{0}+\frac{t}{\tau_{1 / 2}}}{1+\frac{t}{\tau_{1 / 2}}}
\end{aligned}
$$


Here, $\tau_{1 / 2}$ is the half-recovery time and $I_{\infty}$ is the mobile fraction (in eq. $S 7 \mathrm{~b}, I_{\infty}$ is set to 1 ). See Figure $4 d$ for the fitting results.

The diffusion coefficient $(D)$ of the bleached molecule (RGG-EGFP-RGG) can be determined from a 2D or a 3D infinity model, according to eq. S8a or S8b, respectively ${ }^{5}$.

$$
\begin{aligned}
& D=\frac{0.22 \cdot r_{R O I}{ }^{2}}{\tau_{1 / 2}} \\
& D=\frac{0.1 \cdot r_{R O I}{ }^{2}}{\tau_{1 / 2}}
\end{aligned}
$$

Where $r_{\mathrm{ROI}}=1.5 \mu \mathrm{m}$ is the radius of the bleached area, $\tau_{1 / 2}$ is the recovery time from eq. $\mathrm{S} 7$.

The viscosity of RGG condensates was then calculated using the Stokes-Einstein relation (eq. S9) ${ }^{7}$.

$$
\eta=\frac{k_{B} T}{6 \pi R_{H} D}
$$

$R_{\mathrm{H}}$ is the hydrodynamic radius of RGG-EGFP-RGG. Using the online Hydrodynamic Radius Converter (https://www.fluidic.com/resources/Toolkit/hydrodynamic-radius-Converter/), $R_{H}$ was estimated to be $6.54 \mathrm{~nm}$, by taking into consideration the molecular mass and folding of RGGGFP-RGG ${ }^{8}$.

In Figure 4e, the horizontal red dashed line $(\eta=0.8 \mathrm{~Pa} \cdot \mathrm{s})$ represents viscosity calculated assuming the presence of immobile proteins (eq. S7a) in the 2D model (eq. S8a). The vertical red dashed line represents surface tension calculated using $\eta=0.8 \mathrm{~Pa} \cdot \mathrm{s}$ and the higher bound of inverse capillary velocity $(\eta / \gamma=0.018 \mathrm{~s} / \mu \mathrm{m})$ measured from the fusion assay (Figure $4 \mathrm{c})$. The horizontal blue dashed line $(\eta=3.6 \mathrm{~Pa} \cdot \mathrm{s})$ represents viscosity calculated assuming the absence of immobile fraction (eq. S7b) in the 3D model (eq. S8b). The vertical blue dashed line represents surface tension calculated using $\eta=3.6 \mathrm{~Pa} \cdot \mathrm{s}$ and the lower bound of inverse capillary velocity $(\eta / \gamma=0.014 \mathrm{~s} / \mu \mathrm{m})$ measured from the fusion assay (Figure $4 \mathrm{c})$. 


\section{Supplementary Figures}

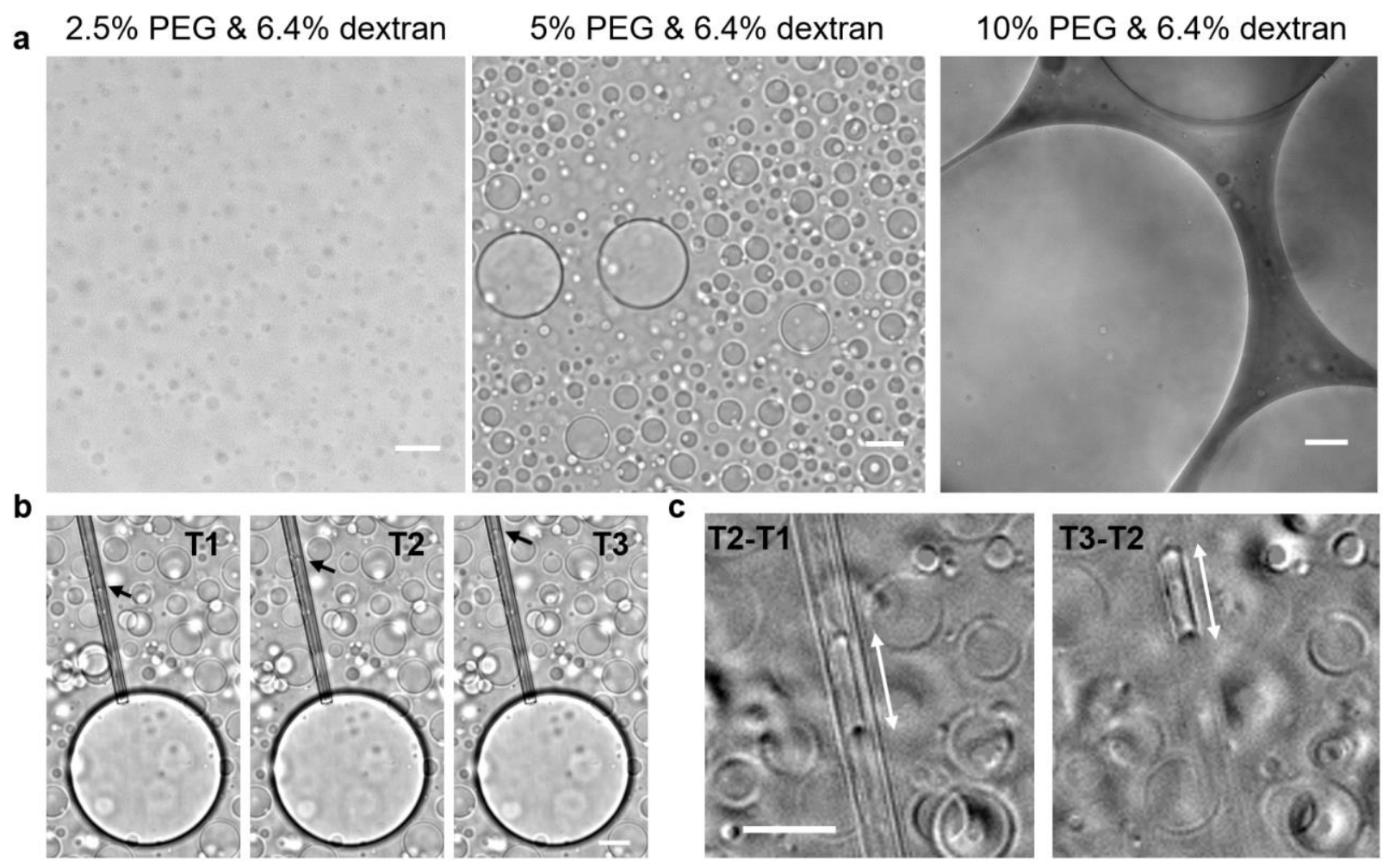

Figure S1: Phase separation and micropipette aspiration analysis of PEG and Dextran mixtures.

a, Micrometer-scale droplets were observed in emulsions of PEG-dextran. Left to right: mixtures of PEG $(8,000 \mathrm{Da})$ and dextran $(500,000 \mathrm{Da})$ at increasing ratios of PEG to dextran. The $5 \%$ PEG \& $6.4 \%$ dextran condition was chosen to produce droplets with similar sizes to those of protein condensates. b. Flow of a dextran-rich condensate into a micropipette (pre-filled with PEG-rich solution) under constant suction pressure (60 Pa). The 3 images were taken at 3 seconds apart. Arrows point to the interfaces between the dextran-rich and PEG-rich phases which are zoomedin in c. c, Intensity differences between images in b: T2-T1 (left) and T3-T2 (right). The doublearrows show the increase of aspiration length in 3 seconds. Analysis of the MPA experiment can be achieved as long as the condensate-buffer interface is resolvable. All scale bars, $20 \mu \mathrm{m}$. 


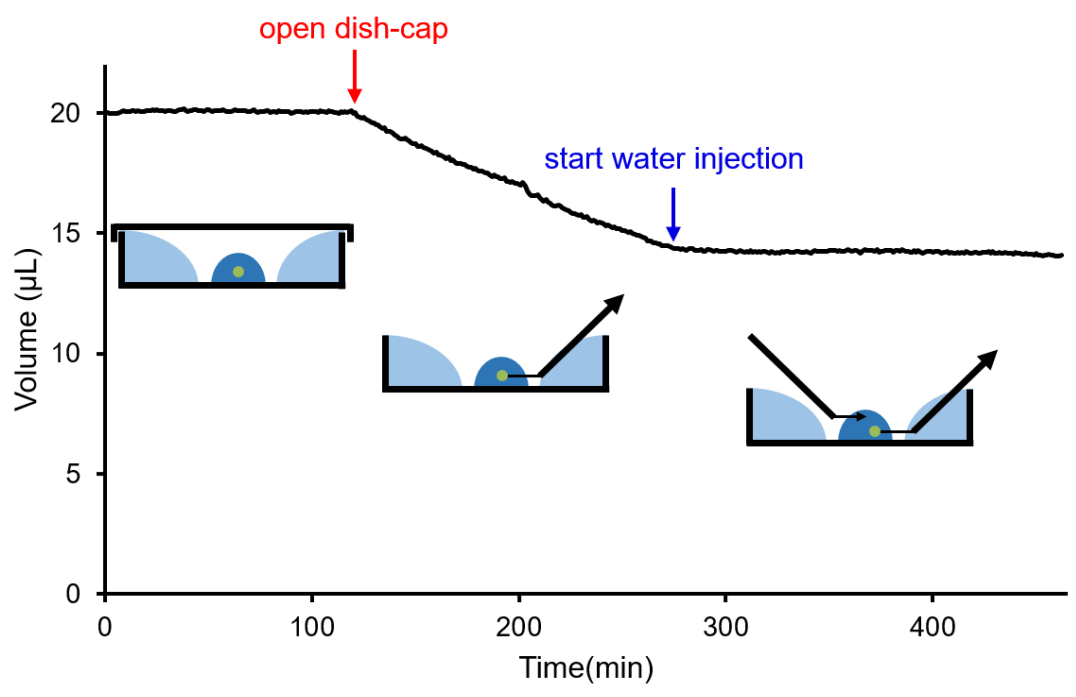

Figure S2: Quantification and correction of water evaporation during micropipette aspiration experiments.

Under our experimental conditions, the presence of peripheral water eliminated evaporation from the $20 \mu \mathrm{L}$ sample as long as the cap of sample dish was on. Upon removing the cap (red arrow) for micropipette aspiration, water slowly evaporated at a rate of $0.04 \mu \mathrm{L} / \mathrm{min}$. The evaporation during micropipette aspiration was compensated (blue arrow) through continuous injection of pure water using a second micropipette, or by adding $2 \mu \mathrm{L}$ of pure water every $50 \mathrm{~min}$. Sample volume was measured through fluorescence-based concentration measurement of Rhodamine-B at an imaging rate of 1 frame per minute and no measurable photobleaching was observed. 


\section{a}

$\begin{array}{llllll}0 \mathrm{~s} & 1 \mathrm{~s} & 2 \mathrm{~s} & 3 \mathrm{~s} & 4 \mathrm{~s} & 5 \mathrm{~s}\end{array}$

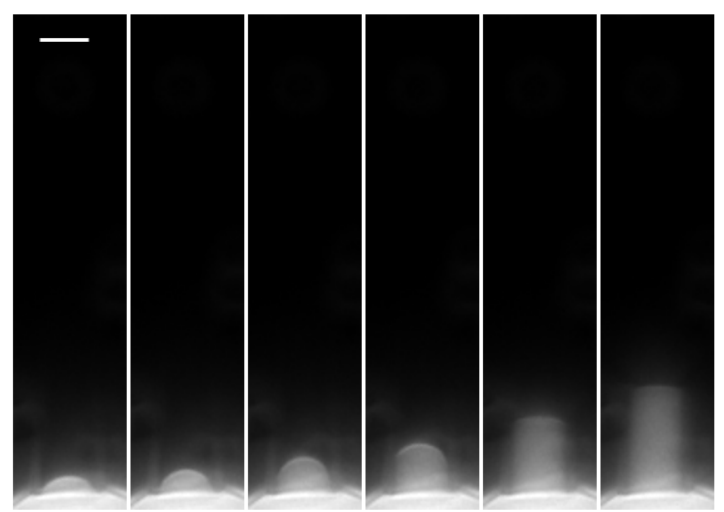

$10 \mathrm{~s}$
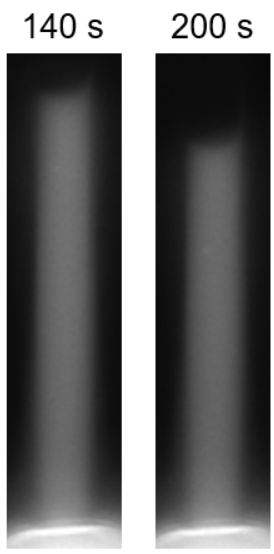

$550 \mathrm{~s}$

$850 \mathrm{~s}$

b

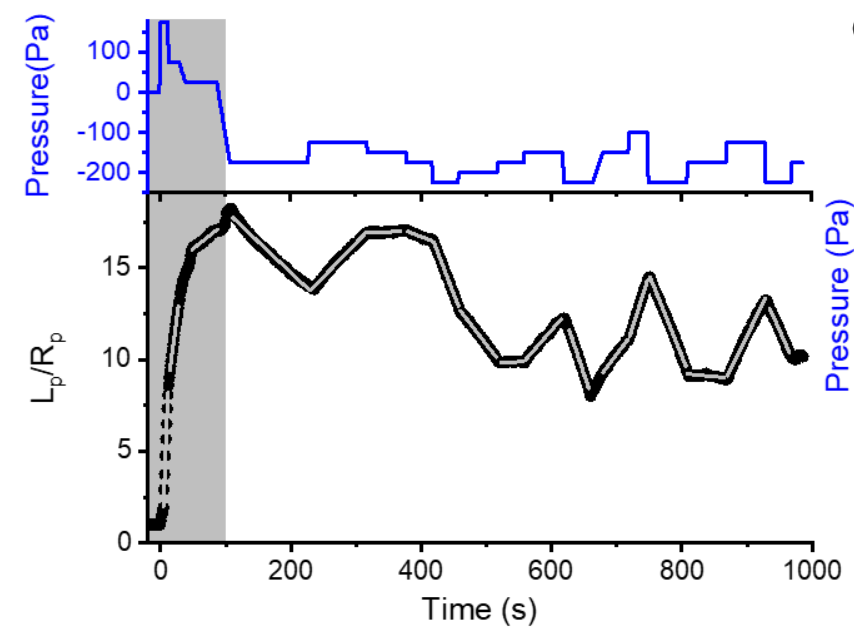

C

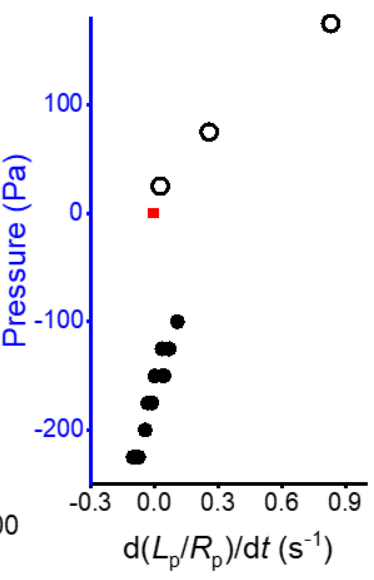

d

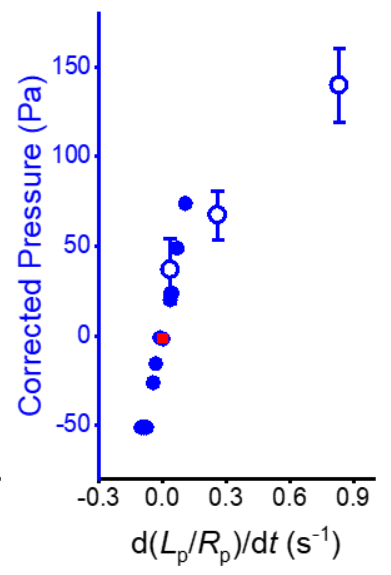

e

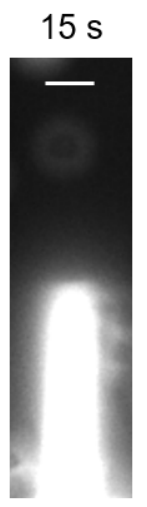

$102 \mathrm{~s}$
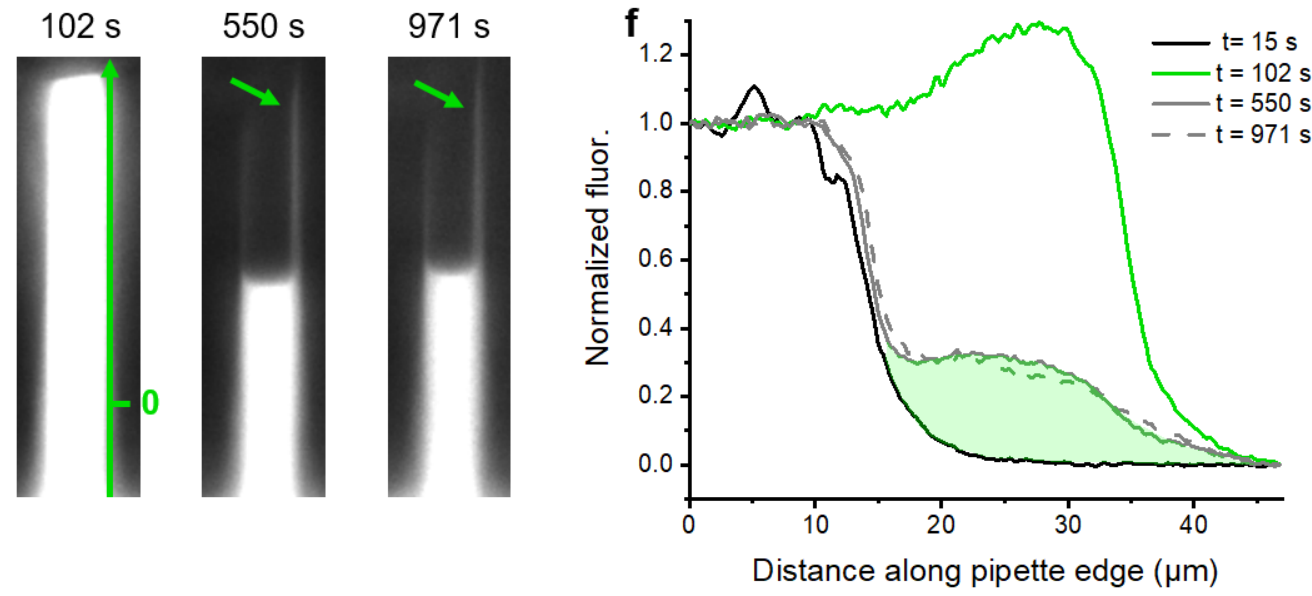

Figure S3: The interfacial curvature and the wetting of RGG condensate inside micropipette.

a, Time lapse fluorescence images showing the aspirated portion of the condensate. After proteins enter the micropipette (1- $4 \mathrm{~s}$ ), the wetting of proteins to the inner pipette wall led to swift changes in the interfacial curvature of the protein condensate. In the case of RGG, this curvature stabilized within 2 min and remained near $-1 / R_{p}$ in the following aspiration steps. $\mathbf{b}$, Aspiration pressure (upper) and normalized aspiration length (lower) as a function of time. Shaded area 
represents the initial-entry steps (defined as when the protein condensate first encountered a bare glass micropipette), where irreversible binding of protein to pipette inner wall happens. Gray lines are linear fits to the normalized aspiration length under each pressure step. c-d, Raw aspiration pressure (c) and tension-corrected pressure (d) of each step plotted against the normalized deformation rate (slopes of the gray lines in $\mathbf{b}$ ). The initial-entry steps are denoted by open circles and a red square is placed at $(0,0)$. Error bars in $\mathbf{d}$ reflect the uncertainty in interfacial curvature during the initial-entry steps. e, Over-exposed images of the aspirated portion during (15 s, $102 \mathrm{~s})$ and after (550 s, $971 \mathrm{~s}$ ) the initial-entry steps. The axis (at $102 \mathrm{~s}$ ) represents the edge of the micropipette, arrow (at $550 \mathrm{~s}$ ) points to proteins that were stuck to the inner pipette wall, which persisted in further aspiration steps (arrow at $971 \mathrm{~s}$ ). $\mathbf{f}$, Line profile along the pipette edge at the four time points shown in e. Area of the shaded region shows the amount of protein that was stuck on the inner wall of the micropipette. All scale bars, $5 \mu \mathrm{m}$. 
a
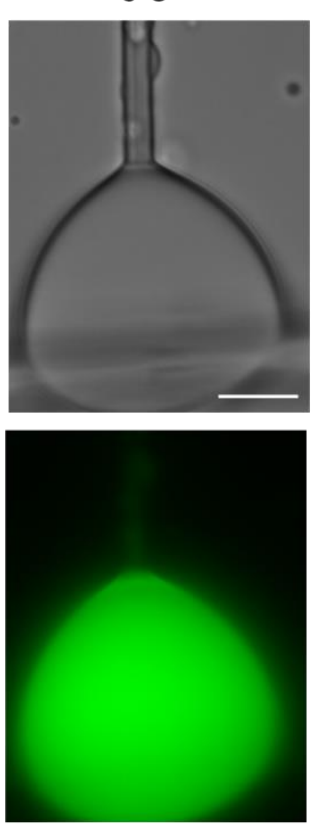

b
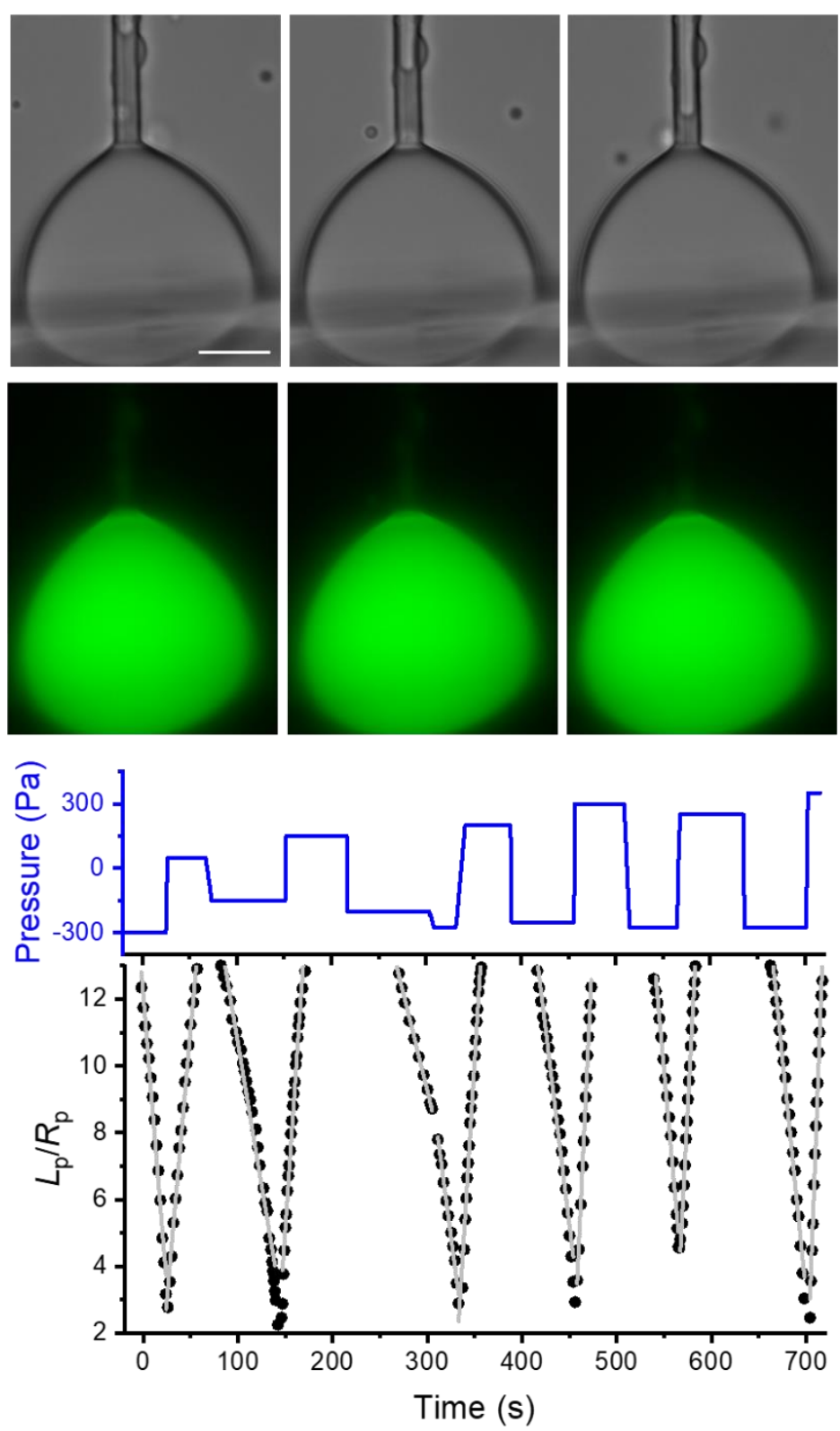

$30 \mathrm{~s}$
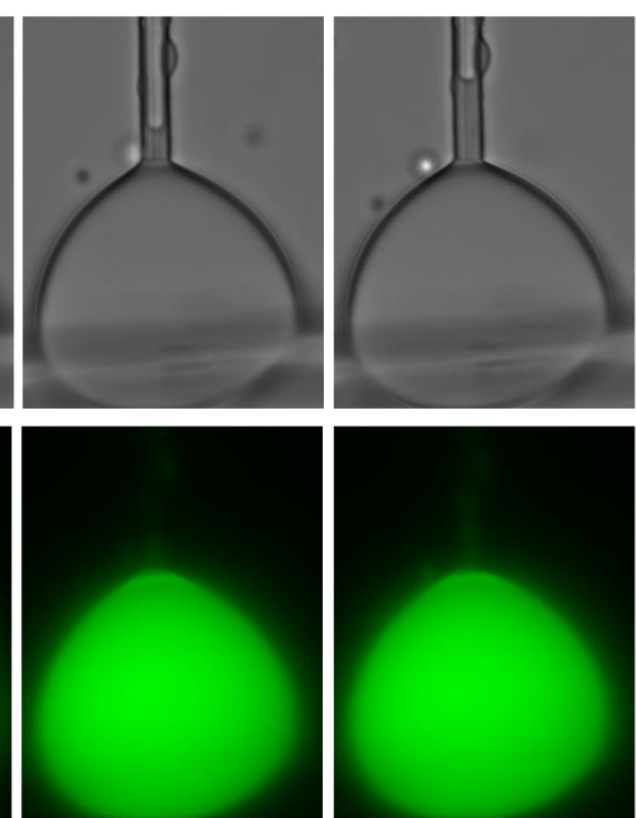

C

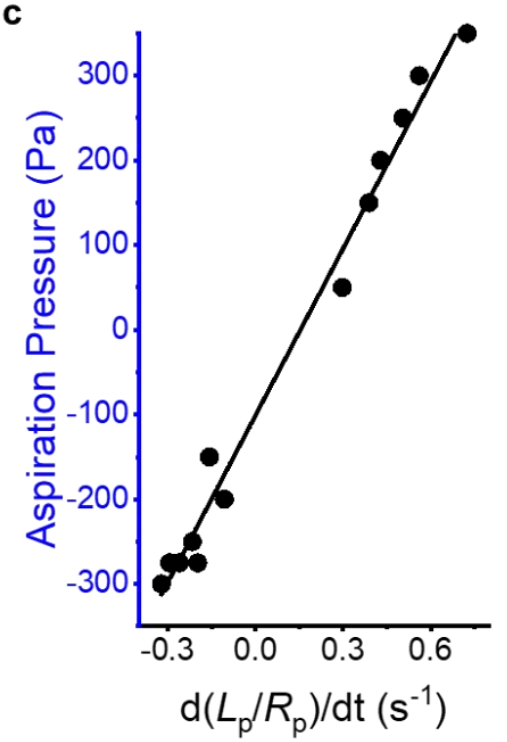

Figure S4: Micropipette aspiration analysis of RGG condensates under transmitted light. a, Time lapse transmitted (upper) and fluorescence (lower) images of an RGG condensate (adhered to a second pipette) under sequential ejection (-300 Pa, 0 20 s) and suction pressures (50 Pa, 20 40 s). b. Aspiration pressure (upper) and normalized aspiration length (lower) quantified from the transmitted light images. Gray lines: linear fits of the normalized aspiration length for each pressure step. c, $P_{\text {asp }}$ of each step plotted against $V$ (slopes of the gray lines in b). The black line represents a linear fit (slope: $660 \pm 30 \mathrm{~Pa} \cdot \mathrm{s}$, intercept: $-100 \pm 10 \mathrm{~Pa}, \mathrm{R}^{2}=0.985$ ). Scale bars, $10 \mu \mathrm{m}$. 
Movie S1: Micropipette aspiration of an RGG condensate free from adhesion to glass surfaces.

Movie S2: Micropipette aspiration of an RGG condensate strongly adhered to a glass pipette.

Movie S3: Micropipette aspiration of an RGG condensate imaged with transmitted light. 


\section{Supplementary Table}

\begin{tabular}{|c|c|c|c|}
\hline Liquid condensate & $\begin{array}{l}\text { Viscosity (Pa.s) } \\
\text { and method }\end{array}$ & $\begin{array}{l}\text { Surface tension } \\
(\mathrm{mN} / \mathrm{m}) \text { and method }\end{array}$ & Note \\
\hline PGL-1 ${ }^{4}$ & $\begin{array}{c}\sim 1 \\
\text { FRAP }\end{array}$ & $\begin{array}{c}\sim 10^{-3} \\
\text { Fusion }\end{array}$ & Order of magnitude estimates \\
\hline LAF-19 ${ }^{9}$ & $\begin{array}{l}23.4 \\
\text { SPT }\end{array}$ & $\begin{array}{l}0.19 \\
\text { Fusion }\end{array}$ & $\begin{array}{l}\text { Average over RNA } \\
\text { concentrations }\end{array}$ \\
\hline Whil3 $^{10}$ & $\begin{array}{c}15 \\
\text { FRAP and SPT }\end{array}$ & $\begin{array}{l}5 \times 10^{-5} \\
\text { Fusion }\end{array}$ & $\begin{array}{l}\text { Average over RNA } \\
\text { concentrations }\end{array}$ \\
\hline NPM1 ${ }^{11}$ & $\begin{array}{c}0.74 \\
\text { FRAP and SPT }\end{array}$ & $\begin{array}{l}8 \times 10^{-4} \\
\text { Fusion and Sessile drop }\end{array}$ & \\
\hline NPM1(in vivo) ${ }^{11}$ & $\begin{array}{c}37 \\
\text { FRAP }\end{array}$ & $\begin{array}{l}\qquad \times 10^{-4} \\
\text { Fusion and Sessile drop }\end{array}$ & \\
\hline FIB1 $^{11}$ & $\begin{array}{c}100 \\
\text { FRAP and SPT }\end{array}$ & $\begin{array}{c}1.23 \times 10^{-3} \\
\text { Fusion and Sessile drop }\end{array}$ & \\
\hline PGL-3 $^{12,13}$ & $\begin{array}{c}1 \text { to } 10^{4} \\
\text { Dual-OT }\end{array}$ & $\begin{array}{c}\sim 4.5 \times 10^{-3} \\
\text { Dual-OT }\end{array}$ & $\begin{array}{l}\text { The viscosity increased } \\
\text { significantly over time }\end{array}$ \\
\hline FUS $^{12}$ & $\begin{array}{l}0.7 \text { to } 50 \\
\text { Dual-OT }\end{array}$ & $\begin{array}{l}\sim 3.1 \times 10^{-3 *} \\
\text { Dual-OT }\end{array}$ & $\begin{array}{l}\text { The viscosity increased } \\
\text { significantly over time }\end{array}$ \\
\hline Poly K ${ }^{14}$ & $\begin{array}{c}0.204 \\
\text { FRAP and SPT }\end{array}$ & $\begin{array}{l}0.017 \\
\text { Fusion }\end{array}$ & \\
\hline Poly $\mathbf{R}^{14}$ & $\begin{array}{c}14.4 \\
\text { FRAP and SPT }\end{array}$ & $\begin{array}{l}0.1 \\
\text { Fusion }\end{array}$ & \\
\hline [RGRGG] $_{5}-\mathrm{dT} 40^{15}$ & $\begin{array}{c}3 \\
\text { SPT and FCS }\end{array}$ & $\begin{array}{c}0.8 \\
\text { Fusion-OT }\end{array}$ & $\begin{array}{c}\text { Average over salt } \\
\text { concentrations }\end{array}$ \\
\hline Dextran-PEG ${ }^{16,17}$ & $\begin{array}{l}0.023 \sim 0.17 \\
\text { Viscometer }\end{array}$ & $\begin{array}{c}0.01 \sim 0.1 \\
\text { Pendant drop }\end{array}$ & $\begin{array}{l}\text { At compositions similar to } \\
\text { sample used in this study }\end{array}$ \\
\hline Olive oil ${ }^{18,19}$ & $\begin{array}{c}0.0741 \\
\text { Viscometer }\end{array}$ & $\begin{array}{c}23.6 \\
\text { Pendent drop }\end{array}$ & \multirow{7}{*}{ All measured in water } \\
\hline Silicon oil20 & $\begin{array}{c}0.02 \\
\text { Viscometer }\end{array}$ & $\begin{array}{c}36 \\
\text { Bubble contour }\end{array}$ & \\
\hline Mineral oil ${ }^{21}$ & $\begin{array}{l}7.75 \times 10^{-3} \\
\text { Viscometer }\end{array}$ & $\begin{array}{c}49 \\
\text { Tensiometer }\end{array}$ & \\
\hline $\mathrm{C}_{16} \mathrm{H}_{34}{ }^{22}$ & $\begin{array}{l}2.77 \times 10^{-3} \\
\text { Viscometer }\end{array}$ & $\begin{array}{c}55.2 \\
\text { Tensiometer }\end{array}$ & \\
\hline $\begin{array}{c}\mathrm{C}_{16} \mathrm{H}_{34} \text { with } \\
\text { surfactants }^{23}\end{array}$ & N.A. & $\begin{array}{l}\text { Lowest to } \sim 0.5 \\
\text { Tensiometer }\end{array}$ & \\
\hline $\mathrm{C}_{10} \mathrm{H}_{22}{ }^{22}$ & $\begin{array}{c}9 \times 10^{-4} \\
\text { Viscometer }\end{array}$ & $\begin{array}{c}53.2 \\
\text { Tensiometer }\end{array}$ & \\
\hline $\mathrm{C}_{6} \mathrm{H}_{14}{ }^{22}$ & $\begin{array}{l}3.13 \times 10^{-4} \\
\text { Viscometer }\end{array}$ & $\begin{array}{c}51.4 \\
\text { Tensiometer }\end{array}$ & \\
\hline
\end{tabular}

* Surface tension value of FUS condensates was kindly provided by Dr. Frank Jülicher via email

FRAP: fluorescence recovery after photobleaching

SPT: single particle tracking

Fusion: first, a ratio of surface tension to viscosity was estimated from the fusion kinetics between two condensates. Then, a separate measure of viscosity was used to calculate surface tension values. Fusion experiments carried out using optical traps were noted as Fusion-OT.

Sessile drop: a prism was used to image condensates of various sizes from the side. The shape is determined by surface tension and gravity of the condensate. A separate measure of condensate density was used to extract surface tension.

Dual-OT: Dual optical traps were used to periodically stretch a condensate via two bead-handles. Viscoelasticity of the condensate was measured via the phase delay of the strain relative to the stress. Surface tension was estimated from the elasticity of condensates. 


\section{Supplementary References}

1. Schuster, B. S.; Reed, E. H.; Parthasarathy, R.; Jahnke, C. N.; Caldwell, R. M.; Bermudez, J. G.; Ramage, H.; Good, M. C.; Hammer, D. A. Controllable protein phase separation and modular recruitment to form responsive membraneless organelles. Nature communications 2018, 9, 1-12.

2. Riedstra, C. P.; McGorty, R. Liquid-Liquid Phase Separation: Undergraduate Labs on a New Paradigm for Intracellular Organization. The Biophysicist 2020, 1.

3. Osterman, N. TweezPal-optical tweezers analysis and calibration software. Comput. Phys. Commun. 2010, 181, 1911-1916.

4. Brangwynne, C. P.; Eckmann, C. R.; Courson, D. S.; Rybarska, A.; Hoege, C.; Gharakhani, J.; Jülicher, F.; Hyman, A. A. Germline P granules are liquid droplets that localize by controlled dissolution/condensation. Science 2009, 324, 1729-1732.

5. Taylor, N. O.; Wei, M.; Stone, H. A.; Brangwynne, C. P. Quantifying dynamics in phaseseparated condensates using fluorescence recovery after photobleaching. Biophys. J. 2019, 117, 1285-1300.

6. Shi, Z.; Graber, Z. T.; Baumgart, T.; Stone, H. A.; Cohen, A. E. Cell membranes resist flow. Cell 2018, 175, 1769-1779. e13.

7. Einstein, A. On the motion of small particles suspended in liquids at rest required by the molecular-kinetic theory of heat. Annalen der physik 1905, 17, 208.

8. Armstrong, J. K.; Wenby, R. B.; Meiselman, H. J.; Fisher, T. C. The hydrodynamic radii of macromolecules and their effect on red blood cell aggregation. Biophys. J. 2004, 87, 42594270.

9. Elbaum-Garfinkle, S.; Kim, Y.; Szczepaniak, K.; Chen, C. C.; Eckmann, C. R.; Myong, S.; Brangwynne, C. P. The disordered P granule protein LAF-1 drives phase separation into droplets with tunable viscosity and dynamics. Proceedings of the National Academy of Sciences 2015, 112, 7189-7194.

10. Zhang, H.; Elbaum-Garfinkle, S.; Langdon, E. M.; Taylor, N.; Occhipinti, P.; Bridges, A. A.; Brangwynne, C. P.; Gladfelter, A. S. RNA controls PolyQ protein phase transitions. Mol. Cell 2015, 60, 220-230.

11. Feric, M.; Vaidya, N.; Harmon, T. S.; Mitrea, D. M.; Zhu, L.; Richardson, T. M.; Kriwacki, R. W.; Pappu, R. V.; Brangwynne, C. P. Coexisting liquid phases underlie nucleolar subcompartments. Cell 2016, 165, 1686-1697.

12. Jawerth, L.; Fischer-Friedrich, E.; Saha, S.; Wang, J.; Franzmann, T.; Zhang, X.; Sachweh, J.; Ruer, M.; ljavi, M.; Saha, S.; Mahamid, J.; Hyman, A. A.; Jülicher, F. Protein condensates as aging Maxwell fluids. Science 2020, 370, 1317-1323. 
13. Jawerth, L. M.; Ijavi, M.; Ruer, M.; Saha, S.; Jahnel, M.; Hyman, A. A.; Jülicher, F.; FischerFriedrich, E. Salt-dependent rheology and surface tension of protein condensates using optical traps. Phys. Rev. Lett. 2018, 121, 258101.

14. Fisher, R. S.; Elbaum-Garfinkle, S. Tunable multiphase dynamics of arginine and lysine liquid condensates. Nature Communications 2020, 11, 1-10.

15. Alshareedah, I.; Thurston, G. M.; Banerjee, P. R. Quantifying viscosity and surface tension of multicomponent protein-nucleic acid condensates. Biophys. J. 2021.

16. Atefi, E.; Mann Jr, J. A.; Tavana, H. Ultralow interfacial tensions of aqueous two-phase systems measured using drop shape. Langmuir 2014, 30, 9691-9699.

17. Carrasco, F.; Chornet, E.; Overend, R. P.; Costa, J. A generalized correlation for the viscosity of dextrans in aqueous solutions as a function of temperature, concentration, and molecular weight at low shear rates. J Appl Polym Sci 1989, 37, 2087-2098.

18. Fisher, L. R.; Mitchell, E. E.; Parker, N. S. Interfacial tensions of commercial vegetable oils with water. J. Food Sci. 1985, 50, 1201-1202.

19. Sahasrabudhe, S. N.; Rodriguez-Martinez, V.; O’Meara, M.; Farkas, B. E. Density, viscosity, and surface tension of five vegetable oils at elevated temperatures: Measurement and modeling. Int. J. Food Prop. 2017, 20, 1965-1981.

20. Peters, F.; Arabali, D. Interfacial tension between oil and water measured with a modified contour method. Colloids Surf. Physicochem. Eng. Aspects 2013, 426, 1-5.

21. Kim, H.; Burgess, D. J. Prediction of interfacial tension between oil mixtures and water. J. Colloid Interface Sci. 2001, 241, 509-513.

22. Goebel, A.; Lunkenheimer, K. Interfacial tension of the water/n-alkane interface. Langmuir 1997, 13, 369-372.

23. Chen, H.; Han, L.; Luo, P.; Ye, Z. The interfacial tension between oil and gemini surfactant solution. Surf. Sci. 2004, 552, L53-L57. 